\title{
Optic nerve head analysis of superior segmental optic hypoplasia using Heidelberg retina tomography
}

This article was published in the following Dove Press journal:

Clinical Ophthalmology

9 October 2010

Number of times this article has been viewed

\author{
Atsushi Miki ${ }^{1,2}$ \\ Motohiro Shirakashi' \\ Kiyoshi Yaoeda' \\ Atsushi Fukushima' \\ Mineo Takagi' \\ Haruki Abe' \\ 'Division of Ophthalmology and \\ Visual Sciences, Niigata University \\ Graduate School of Medical and \\ Dental Sciences, Niigata, ${ }^{2}$ Department \\ of Ophthalmology, Kawasaki Medical \\ School, Okayama, Japan
}

Purpose: To evaluate the optic disc characteristics of eyes with superior segmental optic hypoplasia (SSOH) using the Heidelberg retina tomograph (HRT).

Patients and methods: Thirteen eyes of 13 Japanese patients with SSOH were studied with the HRT (software version: 3.0). The group included six males and seven females, with a mean age of 34.7 years. Six optic disc parameters in the six sectors derived from the patients with SSOH were compared with those of 13 eyes of 13 normal controls. In addition, the diagnostic classification performance of the Frederick S Mikelberg (FSM) discriminant function, glaucoma probability score (GPS), and Moorfields regression analysis (MRA) were assessed.

Results: When compared with normal subjects, many of the optic disc parameters were significantly altered in SSOH in the superior sectors. The area under the curve (AUC) for the receiver operating characteristic was 0.932 for the rim area, 0.926 for the cup-to-disc area ratio, and 0.882 for the cup shape measure. Among the HRT parameters, the largest AUC (0.988) was found for the cup shape measure in the nasal superior segment. The proportion classified as outside normal limits by the FSM discriminant function was $92.3 \%$ (12 eyes). For GPS, six eyes (46.2\%) were classified as outside normal limits. For MRA, when borderline cases were considered test-negative or test-positive, 10 eyes (76.9\%) or 11 eyes (84.6\%) were classified as outside normal limits, respectively. The AUCs were 0.976 for the FSM discriminant function, 0.914 for the MRA overall classification, and 0.710 for the GPS overall classification.

Conclusions: In eyes with $\mathrm{SSOH}$, there is a significant thinning of the rim, especially in the nasal superior sector. Approximately half of the eyes with SSOH were classified as abnormal using indices developed for detecting glaucoma, but the sectorial analysis revealed that the affected sectors were different from those of glaucoma. Optic nerve head measurements using the HRT may be useful in evaluating the optic disc characteristics in eyes with SSOH.

Keywords: superior segmental optic hypoplasia, Heidelberg retina tomography

\section{Introduction}

Superior segmental optic hypoplasia (SSOH) is a congenital optic nerve anomaly characterized by localized inferior visual field defects, superior nerve fiber layer defects, and good visual acuity. ${ }^{1-13}$ The term "superior segmental optic nerve hypoplasia" (SSONH), which is preferred by some investigators, has also sometimes been used instead of $\mathrm{SSOH}$, because the clinical features of this entity include not only a morphologic optic disc anomaly, but also optic nerve fiber defects. ${ }^{10,12} \mathrm{SSOH}$ is considered to be a type of optic nerve hypoplasia, but is unusual in that the central visual acuity is spared. Kim et al have shown four characteristic findings concerning
Correspondence: Atsushi Miki

Department of Ophthalmology, Kawasaki Medical School, 577, Matsushima,

Kurashiki, Okayama 70I-0192, Japan

Tel +8186462 IIII

Fax+8I 864630923

Email amiki@tc5.so-net.ne.jp 
the optic disc, ie, a relative superior entrance of the central retinal artery, a superior peripapillary scleral halo, pallor of the superior disc, and a thinning of the superior peripapillary nerve fiber layer. ${ }^{4}$ However, not all of these features could be consistently found in other reports. ${ }^{6,8-10}$ Instead, a doublering sign, which is a hallmark of optic hypoplasia, has been found. ${ }^{10}$ Also, while previous reports from Western countries have demonstrated that $\mathrm{SSOH}$ is frequently associated with maternal type 1 diabetes mellitus, reports from Japan and Korea have not supported this association. ${ }^{10,12}$ Thus, the definition of $\mathrm{SSOH}$ remains obscure, and it may possibly consist of multiple optic nerve anomalies.

The Heidelberg retina tomograph (HRT), a confocal scanning laser ophthalmoscopy device, has been used to evaluate quantitatively the three-dimensional surface topography of the optic nerve head and the surrounding nerve fiber layer. ${ }^{14-26}$ HRT can provide objective measurements of the optic nerve head, such as the cup-to-disc ratio, cup volume, rim area, and thickness of the nerve fiber layer. It is well known that glaucomatous visual field defects appear only after a substantial number of nerve fibers have been lost. ${ }^{27,28}$ Therefore, HRT is considered to be a promising tool for the early detection of glaucoma. A thinning of the nerve fiber layer often suggests glaucoma, but $\mathrm{SSOH}$ is similar to glaucoma in that nerve fiber layer defects and corresponding visual field losses are observed. ${ }^{8}$ In this study, we investigated the topography of the optic nerve head in SSOH using HRT. This technique includes statistical analyses, Frederick S Mikelberg (FSM) discriminant function, ${ }^{14}$ Moorfields regression analysis (MRA), ${ }^{16}$ and the glaucoma probability score (GPS) classification, ${ }^{18}$ for discriminating glaucomatous and normal optic discs. We also used HRT to evaluate the diagnostic ability of these tests in patients with $\mathrm{SSOH}$.

\section{Subjects and methods}

We examined 13 eyes of 13 patients with SSOH. The mean age \pm standard deviation of the patients was $34.7 \pm 12.9$ (range 15-53) years. There were six males and seven females. Seven had bilateral and six had monocular SSOH. Only one eye was randomly selected and examined for bilateral cases. Each eye had a best-corrected visual acuity $\geq 20 / 20$ and a normal intraocular pressure (IOP) $\leq 21 \mathrm{mmHg}$, with no history of elevated IOP, a normal open angle, and an inferior wedge-shaped visual field defect (oriented to the blind spot) with a corresponding retinal nerve fiber layer defect. The retinal nerve fiber layer defects were detected with ophthalmoscopy and documented by fundus photographs. None of the patients was born of a mother with diabetes mellitus. The average mean deviation of the Humphrey full-threshold 30-2 program was $-4.35 \pm 4.37$ (ranging from -11.96 to +1.76 ) $\mathrm{dB}$. Thirteen eyes of 13 subjects with normal vision were also studied and served as controls. Only one eye was examined for each normal subject. The normal subjects were recruited from the hospital staff or their friends. Ophthalmic examination of these subjects did not reveal any ocular abnormalities.

In each patient, optic disc topography was examined using HRT II (version 3.0; Heidelberg Engineering, GmbH, Heidelberg, Germany). Informed consent was obtained from each subject. HRT II uses a diode laser (670 nm wavelength) to scan the retinal surface sequentially in the horizontal and vertical directions on multiple focal planes. By using confocal scanning principles, a three-dimensional topographic image is constructed from a series of optical image sections at consecutive focal planes. The topographic image determined from the acquired three-dimensional image consists of $384 \times 384(147,456)$ pixels, each of which is a measurement of the surface height of retina and optic nerve head at its corresponding location. For every subject in this study, images were obtained through undilated pupils, with a $15^{\circ}$ field of view. Three topographic images were obtained, combined, and automatically aligned to make a single mean topographic image for analysis. A contour line of the optic disc margin was drawn around the inner margin of the peripapillary scleral ring by an experienced examiner, who had been kept uninformed of the other clinical information. Six HRT parameters obtained with routine analysis were analyzed in terms of the disc area, cup area, cup-to-disc area ratio, rim area, cup shape measure, and height variation contour. Magnification errors were corrected using the subjects' refractive status and corneal-curvature measurements.

Six sectoral (temporal, nasal, superotemporal, superonasal, inferotemporal, and inferonasal) and global optic nerve head parameters were compared between those of the control subjects and those of patients with $\mathrm{SSOH}$, thus producing $P$ values and the area under the curve (AUC for the receiver operating characteristic) for each comparison.

For the FSM discriminant function, the discriminant analysis formula described by Mikelberg et al was used. ${ }^{14}$ We chose the FSM discriminant function because it was thought to be the most representative among such discriminant functions. In the MRA, a measured rim area 
was compared with the predicted rim area adjusted for disc size and age. The normative database was compared in six regions (superior temporal, inferior temporal, temporal, superior nasal, inferior nasal, and nasal) and as an overall global classification. The eyes were classified into three categories: within normal limits (WNL) 95\% confidence intervals (CI); borderline (BL), 95\%-99.9\% CI; and outside normal limits (ONL), 99.9\% CI. In the GPS calculation, parameters describing the shape of the optic nerve head and retinal nerve fiber layer were calculated based on the mathematical model derived from normal and glaucomatous eyes in HRT. The parameters thus obtained were used to compute the GPS numeric scores. Then, the final GPS was used for the classification was WNL $(0 \%-27 \%)$, BL (28\%-64\%), and ONL (65\%-100\%). The AUC curves were plotted between the normal and SSOH eyes for the FSM discriminant function, MRA, and GPS. The categoric variable was computed where "normal" $=0$, "borderline" $=1$, and "outside normal limits" $=2$.

We used Mann-Whitney's $U$ test for each comparison. Because of the large number of tests, the Bonferroni correction for multiple comparisons was also applied. SPSS (17.0 J; SPSS Japan, Inc., Tokyo, Japan) and MedCalc (version 10.1; MedCalc Software bvba, Mariakerke, Belgium) were used to perform the statistical analysis.

\section{Results}

Table 1 shows the demographic and clinical characteristics of the subjects included in the study. Gender, age, and refraction did not differ significantly between the patients with $\mathrm{SSOH}$ and normal subjects. None of the patients with SSOH showed a progression of the visual field defects in the

Table I Demographic and clinical characteristics of the subjects included in the study

\begin{tabular}{llll}
\hline & SSOH $(\mathbf{N}=13) *$ & Normal $(\mathbf{N}=13)^{*}$ & $\boldsymbol{P}_{\text {value }}^{\dagger}$ \\
\hline Sex (M/F) & $6 / 7$ & $4 / 9$ & 0.420 \\
Age (years) & $34.7 \pm 12.9$ & $34.6 \pm 9.1$ & 0.840 \\
& $(15,53)$ & $(22,55)$ & \\
Refraction & $-3.7| \pm 3.3|$ & $-3.58 \pm 1.92$ & 0.614 \\
(diopters) & $(-10.00,-0.50)$ & $(-7.25,-1.13)$ & \\
MD (dB) & $-4.35 \pm 4.37$ & $1.26 \pm 1.04$ & $<0.001$ \\
& $(-11.96,+1.76)$ & $(-0.88,+2.92)$ & \\
\hline
\end{tabular}

Notes: *Data are presented as mean \pm standard deviation (range); ${ }^{\dagger} P$ value is for comparison between $\mathrm{SSOH}$ and normal eyes using Mann-Whitney's $U$ test; ${ }^{\ddagger}$ mean deviation of Humphrey 30-2 visual field.

Abbreviations: $\mathrm{SSOH}$, superior segmental optic hypoplasia; $\mathrm{M}$, male; F, female; MD, mean deviation. subsequent follow-up. The follow-up period was $74.4 \pm 27.2$ (range 48-133) months.

Table 2 shows various HRT parameters of the patients with $\mathrm{SSOH}$ and the healthy control subjects. There was no statistically significant difference between the disc area of patients with SSOH and normal subjects. The cup area and cup-to-disc area ratio were larger, while the rim area was reduced, in patients with $\mathrm{SSOH}$, especially in the superonasal area. The parameters with the largest AUCs were the nasal superior cup shape measure (0.988), the nasal superior cupto-disc area ratio $(0.970)$, and the nasal superior rim area (0.959). Figure 1 shows the AUC for the nasal superior cup shape measure.

The results of the diagnostic classification tests are shown in Table 3. Using the FSM discriminant function, $92.3 \%$ of the eyes were classified as outside the normal limits. By the MRA and GPS, $84.6(* 76.9) \%$ and $46.2 \%$ of the eyes were classified as abnormal, respectively (when the borderline was classified as normal). The MRA in each sector revealed that the superonasal rim area was most frequently abnormal (Figure 2). The AUC was 0.976 for the FSM discriminant function, 0.914 for the MRA, and 0.710 for the GPS. The sectoral analysis of MRA revealed the largest AUC (0.956) in the nasal superior sector. The FSM discriminant function showed a sensitivity of $92.3 \%$ and a specificity of $92.3 \%$. The MRA overall classification had a sensitivity of 84.6 (76.9)\% and a specificity of $92.3(100) \%$. The GPS overall classification had a sensitivity of 46.2 (46.2)\% and a specificity of $92.3(100) \%$.

\section{Discussion}

Our sectorial analysis of the optic nerve head has shown that many of the parameters differed significantly between normal subjects and patients with SSOH. Also, a large proportion of the eyes with $\mathrm{SSOH}$ were determined to be abnormal using the FSM discriminant function and MRA. It is important to distinguish this condition from glaucoma, because costly antiglaucoma medication is not warranted in the presence of $\mathrm{SSOH}$, assuming that $\mathrm{SSOH}$ is a nonprogressive disease. Whereas a notch in the inferotemporal or superotemporal rim is commonly found in glaucoma, the rim area in the superonasal segment was found by this study to be most decreased in cases of SSOH. Our results may be generalized to cases of $\mathrm{SSOH}$ in general, because it is thought to be a nonprogressive disease and the degree of disease severity is not so variable as in glaucoma. ${ }^{21,24}$ There may be "subclinical" $\mathrm{SSOH}$ in which visual field testing does not detect any abnormality, 
Table 2 Global and segmental optic disc parameters and areas under the receiver operating characteristic curve for each parameter

\begin{tabular}{|c|c|c|c|c|}
\hline & SSOH $(\mathbf{N}=13)^{*}$ & Normal $(\mathbf{N}=13)^{*}$ & $P$ value & AUC $\ddagger$ \\
\hline DA global & $1.97 \pm 0.55(1.18-3.17)$ & $2.05 \pm 0.47(1.12-2.86)$ & 0.650 & $0.556(0.350-0.749)$ \\
\hline DA temporal & $0.48 \pm 0.15(0.24-0.81)$ & $0.47 \pm 0.11(0.25-0.63)$ & 0.920 & $0.515(0.313-0.714)$ \\
\hline DA TS & $0.26 \pm 0.05(0.19-0.36)$ & $0.29 \pm 0.07(0.18-0.43)$ & 0.243 & $0.636(0.426-0.814)$ \\
\hline DA TI & $0.25 \pm 0.07(0.15-0.40)$ & $0.28 \pm 0.08(0.15-0.45)$ & 0.287 & $0.624(0.4 \mid 4-0.804)$ \\
\hline DA nasal & $0.47 \pm 0.15(0.24-0.79)$ & $0.48 \pm 0.12(0.24-0.64)$ & 0.801 & $0.533(0.328-0.729)$ \\
\hline DA NS & $0.25 \pm 0.08(0.14-0.44)$ & $0.26 \pm 0.07(0.13-0.39)$ & 0.579 & $0.568(0.36 \mathrm{I}-0.759)$ \\
\hline DA NI & $0.26 \pm 0.06(0.20-0.39)$ & $0.27 \pm 0.06(0.18-0.37)$ & 0.311 & $0.621(0.411-0.802)$ \\
\hline CA global & $0.93 \pm 0.51(0.29-1.98)$ & $0.40 \pm 0.22(0.03-0.78)$ & 0.007 & $0.805(0.603-0.932)$ \\
\hline CA temporal & $0.28 \pm 0.16(0.08-0.58)$ & $0.19 \pm 0.10(0.02-0.32)$ & 0.153 & $0.666(0.455-0.837)$ \\
\hline CA TS & $0.14 \pm 0.06(0.05-0.23)$ & $0.06 \pm 0.04(0.00-0.11)$ & $<\mathbf{0 . 0 0 1}$ & $0.882(0.694-0.973)$ \\
\hline CA TI & $0.09 \pm 0.07(0.00-0.21)$ & $0.04 \pm 0.03(0.00-0.09)$ & 0.050 & $0.728(0.519-0.882)$ \\
\hline CA nasal & $0.21 \pm 0.15(0.00-0.54)$ & $0.06 \pm 0.05(0.00-0.16)$ & 0.003 & 0.83 I (0.634-0.947) \\
\hline CA NS & $0.15 \pm 0.06(0.02-0.28)$ & $0.04 \pm 0.03(0.00-0.11)$ & $<0.001$ & $0.932(0.760-0.991)$ \\
\hline CA NI & $0.07 \pm 0.05(0.00-0.15)$ & $0.02 \pm 0.01(0.00-0.04)$ & 0.002 & $0.843(0.648-0.954)$ \\
\hline RA global & $1.04 \pm 0.18(0.78-1.38)$ & $1.66 \pm 0.45(1.03-2.53)$ & $<0.001$ & $0.932(0.760-0.991)$ \\
\hline RA temporal & $0.20 \pm 0.05(0.09-0.30)$ & $0.28 \pm 0.11(0.18-0.52)$ & 0.019 & $0.769(0.563-0.910)$ \\
\hline RA TS & $0.12 \pm 0.03(0.07-0.18)$ & $0.23 \pm 0.07(0.1 \mathrm{I}-0.33)$ & $<0.001$ & $0.926(0.752-0.989)$ \\
\hline RA TI & $0.16 \pm 0.04(0.09-0.25)$ & $0.24 \pm 0.08(0.14-0.43)$ & 0.002 & $0.843(0.648-0.954)$ \\
\hline RA nasal & $0.26 \pm 0.08(0.10-0.38)$ & $0.42 \pm 0.11(0.21-0.62)$ & $<0.001$ & $0.891(0.706-0.977)$ \\
\hline RA NS & $0.10 \pm 0.03(0.04-0.16)$ & $0.22 \pm 0.07(0.13-0.33)$ & $<0.001$ & $0.959(0.799-0.994)$ \\
\hline RA NI & $0.20 \pm 0.05(0.14-0.28)$ & $0.26 \pm 0.06(0.18-0.37)$ & 0.006 & $0.811(0.610-0.935)$ \\
\hline CDAR global & $0.45 \pm 0.14(0.23-0.66)$ & $0.19 \pm 0.10(0.01-0.35)$ & $<0.001$ & $0.926(0.752-0.989)$ \\
\hline CDAR temporal & $0.55 \pm 0.19(0.24-0.82)$ & $0.40 \pm 0.18(0.06-0.63)$ & 0.072 & $0.710(0.500-0.869)$ \\
\hline CDAR TS & $0.52 \pm 0.15(0.24-0.78)$ & $0.21 \pm 0.15(0.01-0.47)$ & $<\mathbf{0 . 0 0 1}$ & $0.929(0.756-0.990)$ \\
\hline CDAR TI & $0.32 \pm 0.21(0.01-0.67)$ & $0.14 \pm 0.11(0.00-0.27)$ & 0.026 & $0.754(0.547-0.900)$ \\
\hline CDAR nasal & $0.4 \mathrm{I} \pm 0.22(0.00-0.68)$ & $0.12 \pm 0.10(0.00-0.32)$ & 0.001 & $0.86 \mathrm{I}(0.669-0.963)$ \\
\hline CDAR NS & $0.58 \pm 0.14(0.17-0.74)$ & $0.15 \pm 0.12(0.00-0.43)$ & $<\mathbf{0 . 0 0 1}$ & $0.970(0.817-0.992)$ \\
\hline CDAR NI & $0.24 \pm 0.15(0.00-0.48)$ & $0.06 \pm 0.06(0.00-0.16)$ & 0.001 & $0.855(0.662-0.960)$ \\
\hline HVC & $0.39 \pm 0.13(0.18-0.68)$ & $0.41 \pm 0.07(0.27-0.52)$ & 0.264 & $0.633(0.423-0.81 \mathrm{I})$ \\
\hline HVC temporal & $0.15 \pm 0.06(0.04-0.25)$ & $0.20 \pm 0.06(0.12-0.34)$ & 0.101 & $0.692(0.482-0.856)$ \\
\hline HVC TS & $0.16 \pm 0.15(0.02-0.48)$ & $0.17 \pm 0.06(0.05-0.29)$ & 0.186 & $0.654(0.443-0.828)$ \\
\hline HVC TI & $0.21 \pm 0.08(0.10-0.38)$ & $0.23 \pm 0.09(0.09-0.38)$ & 0.579 & $0.568(0.36 \mathrm{I}-0.759)$ \\
\hline HVC nasal & $0.14 \pm 0.07(0.06-0.27)$ & $0.11 \pm 0.04(0.06-0.23)$ & 0.479 & $0.586(0.378-0.774)$ \\
\hline HVC NS & $0.09 \pm 0.04(0.03-0.18)$ & $0.05 \pm 0.02(0.03-0.11)$ & 0.019 & $0.769(0.563-0.910)$ \\
\hline HVC NI & $0.09 \pm 0.05(0.02-0.24)$ & $0.07 \pm 0.04(0.02-0.15)$ & 0.186 & $0.654(0.443-0.828)$ \\
\hline CSM & $-0.08 \pm 0.10(-0.28-0.11)$ & $-0.21 \pm 0.05(-0.28,-0.10)$ & $<0.001$ & $0.882(0.694-0.973)$ \\
\hline CSM temporal & $-0.07 \pm 0.08(-0.19-0.09)$ & $-0.11 \pm 0.06(-0.22,-0.01)$ & 0.113 & $0.686(0.476-0.852)$ \\
\hline CSM TS & $0.05 \pm 0.09(-0.08-0.25)$ & $-0.14 \pm 0.12(-0.31-0.03)$ & $<0.001$ & $0.905(0.725-0.983)$ \\
\hline CSM TI & $-0.07 \pm 0.14(-0.37-0.16)$ & $-0.22 \pm 0.10(-0.39,-0.07)$ & 0.006 & $0.811(0.610-0.935)$ \\
\hline CSM nasal & $-0.00 \pm 0.15(-0.25-0.30)$ & $-0.18 \pm 0.13(-0.46,-0.02)$ & 0.005 & $0.814(0.613-0.937)$ \\
\hline CSM NS & $0.15 \pm 0.15(-0.13-0.40)$ & $-0.18 \pm 0.08(-0.33,-0.03)$ & $<0.001$ & $0.988(0.845-1.000)$ \\
\hline CSM NI & $-0.10 \pm 0.18(-0.40-0.18)$ & $-0.31 \pm 0.15(-0.55,-0.04)$ & 0.006 & $0.808(0.606-0.934)$ \\
\hline
\end{tabular}

Notes: *Data are presented as mean \pm standard deviation (range); ${ }^{\dagger} P$ value is for comparison of the parameters between SSOH and normal eyes using Mann-Whitney's $U$ test. Because of the multiple comparisons, the Bonferroni correction was applied, with a level of significance of 0.00119 . Bold $P$ values are statistically significant. ${ }^{ \pm}$Data are expressed with $95 \%$ confidence intervals of each value in parentheses.

Abbreviations: DA, disc area; CA, cup area; RA, rim area; CDAR, cup-to-disc area ratio; HVC, height variation contour; CSM, cup shape measure; TS, temporal superior; TI, temporal inferior; NS, nasal superior; NI, nasal inferior.

while, in fact, nerve fibers are already reduced. HRT could be helpful in such cases.

The definition of SSOH remains to be determined. So far, different studies have used different criteria to define $\mathrm{SOOH}$. We used diagnostic criteria based on a previous report. ${ }^{10}$ However, the appearances of the optic discs of $\mathrm{SSOH}$ in this study appear to be somewhat different from those reported in Western countries. In general, the optic discs of SSOH in Japan often have a glaucoma-like cupping. ${ }^{7,8,10}$ Although glaucoma has to be excluded in the initial diagnosis of $\mathrm{SSOH}$, because the former is an acquired optic nerve disorder, the association between $\mathrm{SSOH}$ and 
Nasal superior cup shape measure

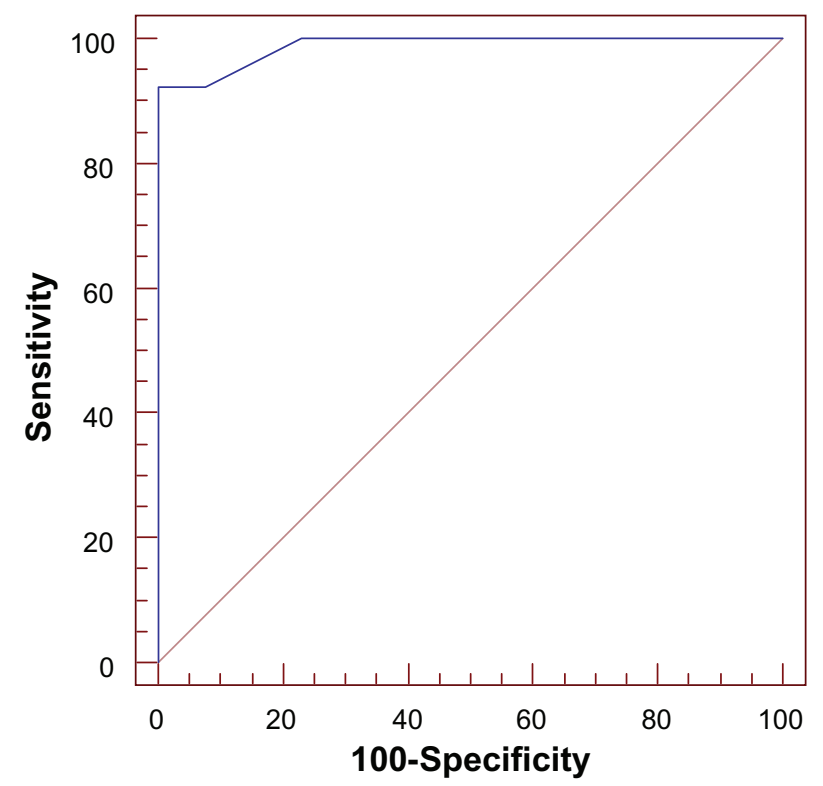

Figure I A receiver operating characteristic curve for the nasal superior cup shape measure.

glaucoma remains unclear. It is sometimes difficult to differentiate $\mathrm{SSOH}$ from glaucoma (especially from normal-tension glaucoma, in which the IOP is within normal limits) because the two share similar optic disc appearance features, such as a large cupping and a rim thinning. ${ }^{10}$ Furthermore, glaucoma and $\mathrm{SSOH}$ do coexist in some cases. However, optic discs in the case reports from Western countries do not seem to show any general glaucoma-like cupping. ${ }^{11}$ Additionally, SSOH is almost never associated with maternal diabetes mellitus in Japan. Thus, SSOH may be heterogeneous, and our conclusion may apply only to the specific type of SSOH that is typically found in Japan or Korea. However, superior nerve fiber layer defects and corresponding inferior visual field defects (either inferior altitudinal defects or inferior sector defects connected to the blind spot) are always observed in $\mathrm{SSOH}$.

The visual fields in $\mathrm{SSOH}$ are shown to be stable over a 10 -year period ${ }^{9}$ or an eight-year period, ${ }^{10}$ but the long-term prognosis is not known, because no longer follow-ups have yet been reported. SSOH has been found in young patients, in whom glaucoma is less likely than in aged patients. ${ }^{10}$ These findings seem to support the notion that $\mathrm{SSOH}$ is a congenital anomaly and does not progress. HRT could be used for the patients with $\mathrm{SSOH}$ over time to make sure that there is no progression of the disease.

\section{Nasal superior MRA}

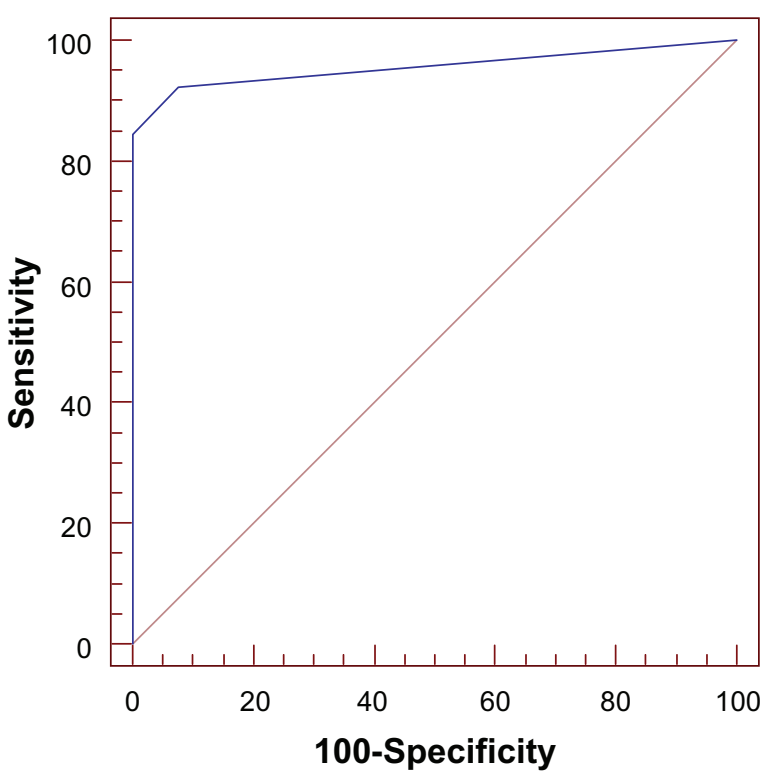

Figure 2 A receiver operating characteristic curve for the nasal superior Moorfields regression analysis (MRA).

Subjective qualitative techniques, such as ophthalmoscopy, are dependent on the examiner's experience. However, only a few previous reports investigating SSOH have utilized objective quantitative imaging technologies of the optic disc. ${ }^{7,10,11}$ Unoki et al used OCT to investigate the tomography of the optic disc and peripapillary retina in patients with SSOH. ${ }^{7}$ Using OCT, obviously abnormal findings may be observed in patients in whom the optic disc shows only minimal changes, even in the fundus photograph. ${ }^{7}$ Although both OCT and HRT can provide objective quantitative measurements of optic disc topography, the fundamental measurement principles of these two instruments are different. HRT is thought to be specifically useful for accurate structural assessments of the optic nerve head. ${ }^{16,19}$ A recent study utilizing OCT and HRT in SSOH generally supports our findings. ${ }^{13}$

In this study, most eyes with $\mathrm{SSOH}$ were classified as abnormal, with a high specificity, using the FSM discriminant function and MRA. HRT generally requires a manual drawing of the contour line of the edge of the optic disc, ${ }^{16,19}$ and this process introduces an element of uncontrolled variability into the analysis because such contour lines, drawn by different observers, may vary considerably. The outlining of the disc margin is not necessary for GPS, and thus GPS is considered to be a more objective method than MRA. ${ }^{18}$ While sensitivity and specificity values were found to be comparable between MRA and GPS in some reports, ${ }^{20}$ the 
Table 3 ROC, sensitivity, and specificity for the FSM discriminant function, MRA, and GPS used to discriminate between normal eyes and eyes with $\mathrm{SSOH}$

\begin{tabular}{|c|c|c|c|c|}
\hline & ROC & & Sensitivity & Specificity \\
\hline FSM & $0.976(0.826-0.990)$ & & $92.3(63.9-98.7) \%$ & $92.3(63.9-98.7) \%$ \\
\hline \multirow[t]{2}{*}{ MRA overall } & $0.914(0.736-0.986)$ & $\mathrm{BL}=$ abnormal & $84.6(54.5-97.6) \%$ & $92.3(63.9-98.7) \%$ \\
\hline & & $\mathrm{BL}=$ normal & $76.9(46.2-94.7) \%$ & $100(75.1-100) \%$ \\
\hline \multirow[t]{2}{*}{ MRA global } & $0.885(0.698-0.974)$ & $\mathrm{BL}=$ abnormal & $76.9(46.2-94.7) \%$ & $100(75.1-100) \%$ \\
\hline & & $\mathrm{BL}=$ normal & $76.9(46.2-94.7) \%$ & $100(75.1-100) \%$ \\
\hline \multirow[t]{2}{*}{ MRA temporal } & $0.538(0.334-0.734)$ & $\mathrm{BL}=$ abnormal & $7.7(1.3-36.1) \%$ & $100(75.1-100) \%$ \\
\hline & & $\mathrm{BL}=$ normal & $0.0(0.0-24.9) \%$ & $100(75.1-100) \%$ \\
\hline \multirow[t]{2}{*}{ MRA temporal superior } & $0.654(0.443-0.828)$ & $\mathrm{BL}=$ abnormal & $30.8(9.3-61.4) \%$ & $100(75.1-100) \%$ \\
\hline & & $\mathrm{BL}=$ normal & $30.8(9.3-61.4) \%$ & $100(75.1-100) \%$ \\
\hline \multirow[t]{2}{*}{ MRA temporal inferior } & $0.615(0.406-0.797)$ & $\mathrm{BL}=$ abnormal & $23.1(5.3-53.8) \%$ & $100(75.1-100) \%$ \\
\hline & & $\mathrm{BL}=$ normal & $23.1(5.3-53.8) \%$ & $100(75.1-100) \%$ \\
\hline \multirow[t]{2}{*}{ MRA nasal } & $0.846(0.65 \mathrm{I}-0.955)$ & $\mathrm{BL}=$ abnormal & $69.2(38.6-90.7) \%$ & $100(75.1-100) \%$ \\
\hline & & $\mathrm{BL}=$ normal & $69.2(38.6-90.7) \%$ & $100(75.1-100) \%$ \\
\hline \multirow[t]{2}{*}{ MRA nasal superior } & $0.956(0.794-0.994)$ & $\mathrm{BL}=$ abnormal & $92.3(63.9-98.7) \%$ & $92.3(63.9-98.7) \%$ \\
\hline & & $\mathrm{BL}=$ normal & $84.6(54.5-97.6) \%$ & $100(75.1-100) \%$ \\
\hline \multirow[t]{2}{*}{ MRA nasal inferior } & $0.654(0.443-0.828)$ & $\mathrm{BL}=\mathrm{abnormal}$ & $30.8(9.3-61.4) \%$ & $100(75.1-100) \%$ \\
\hline & & $\mathrm{BL}=$ normal & $30.8(9.3-61.4) \%$ & $100(75.1-100) \%$ \\
\hline \multirow[t]{2}{*}{ GPS overall } & $0.710(0.500-0.869)$ & $\mathrm{BL}=\mathrm{abnormal}$ & $46.2(19.3-74.8) \%$ & $92.3(63.9-98.7) \%$ \\
\hline & & $\mathrm{BL}=$ normal & $46.2(19.3-74.8) \%$ & $100(75.1-100) \%$ \\
\hline \multirow[t]{2}{*}{ GPS global } & $0.731(0.522-0.884)$ & $\mathrm{BL}=$ abnormal & $46.2(19.3-74.8) \%$ & $100(75.1-100) \%$ \\
\hline & & $\mathrm{BL}=$ normal & $46.2(19.3-74.8) \%$ & $100(75.1-100) \%$ \\
\hline \multirow[t]{2}{*}{ GPS temporal } & $0.727(0.509-0.886)$ & $\mathrm{BL}=$ abnormal & 45.5 (16.9-76.5)\% & $100(75.1-100) \%$ \\
\hline & & $\mathrm{BL}=$ normal & 45.5 (I6.9-76.5)\% & $100(75.1-100) \%$ \\
\hline \multirow[t]{2}{*}{ GPS temporal superior } & $0.706(0.48-0.872)$ & $\mathrm{BL}=$ abnormal & $45.5(16.9-76.5) \%$ & $92.3(63.9-98.7) \%$ \\
\hline & & $\mathrm{BL}=$ normal & $45.5(16.9-76.5) \%$ & $100(75.1-100) \%$ \\
\hline \multirow[t]{2}{*}{ GPS temporal inferior } & $0.727(0.509-0.886)$ & $\mathrm{BL}=$ abnormal & 45.5 (16.9-76.5)\% & $100(75.1-100) \%$ \\
\hline & & $\mathrm{BL}=$ normal & $45.5(16.9-76.5) \%$ & $100(75.1-100) \%$ \\
\hline \multirow[t]{2}{*}{ GPS nasal } & $0.727(0.509-0.886)$ & $\mathrm{BL}=\mathrm{abnormal}$ & $45.5(16.9-76.5) \%$ & $100(75.1-100) \%$ \\
\hline & & $\mathrm{BL}=$ normal & $45.5(16.9-76.5) \%$ & $100(75.1-100) \%$ \\
\hline \multirow[t]{2}{*}{ GPS nasal superior } & $0.727(0.509-0.886)$ & $\mathrm{BL}=\mathrm{abnormal}$ & $45.5(16.9-76.5) \%$ & $100(75.1-100) \%$ \\
\hline & & $\mathrm{BL}=$ normal & $45.5(16.9-76.5) \%$ & $100(75.1-100) \%$ \\
\hline \multirow[t]{2}{*}{ GPS nasal inferior } & $0.727(0.509-0.886)$ & $\mathrm{BL}=$ abnormal & $45.5(16.9-76.5) \%$ & $100(75.1-100) \%$ \\
\hline & & $\mathrm{BL}=$ normal & $45.5(16.9-76.5) \%$ & $100(75.1-100) \%$ \\
\hline
\end{tabular}

Note: Data are expressed with $95 \%$ confidence intervals of each value in the parentheses.

Abbreviations: FSM, FSM classification; MRA, Moorfield regression analysis; GPS, glaucoma probability score; "BL = abnormal", when the borderline is regarded as abnormal; "BL = normal", when the borderline is regarded as normal; ROC, receiver operating characteristic; SSOH; superior segmental optic hypoplasia.

agreement between MRA and GPS is not necessarily high in defining glaucoma in other reports. ${ }^{22}$ This study also shows that such an agreement is not high in $\mathrm{SSOH}$ either, probably reflecting the differences in the analysis algorithm. It is not surprising to find a rather low sensitivity for the GPS, because this is a diagnostic test designed specifically for glaucoma, ${ }^{18}$ but not for SSOH. Alternatively, the GPS results in this study may have revealed that the GPS is not sensitive to the local rim-volume losses, which are shown by MRA, because of the influence of neighboring normal sectors. ${ }^{20}$ Therefore, sectorial HRT-GPS analysis appears to have some limitations, although it has the clear merit that it is independent of the contour line traced manually by an examiner. Although it has been reported that both GPS and MRA are likely to produce many false-positive classifications in glaucoma, ${ }^{20}$ high specificity values were obtained in SSOH. It has been reported that optic disc size influences the diagnostic accuracy of HRT in glaucoma, with small optic discs showing a lower sensitivity and a higher specificity compared with larger discs. ${ }^{24}$ In this study, the disc area in SSOH was not statistically significantly different from that of the controls.

Since diagnostic tests for glaucoma using HRT frequently determine the $\mathrm{SSOH}$ to be abnormal, it will be necessary in a future study to develop a test which can reliably differentiate SSOH from glaucoma. From our results, it seems that HRT can discern the pattern of the area of rim losses between SSOH and glaucoma. Our results also indicate that HRT can be useful in identifying a reduction of the rim area, especially in the superonasal region, in patients with $\mathrm{SSOH}$. 


\section{Disclosure}

This research was presented at the World Glaucoma Congress in Boston, USA, July 8-11, 2009. The authors report no conflicts of interest in this work.

\section{References}

1. Gardner HB, Irvine AR. Optic nerve hypoplasia with good visual acuity. Arch Ophthalmol. 1972;88:255-258.

2. Petersen RA, Walton DS. Optic nerve hypoplasia with good visual acuity and visual field defects: A study of children of diabetic mothers. Arch Ophthalmol. 1977;95:254-258.

3. Björk A, Laurell CG, Laurell U. Bilateral optic nerve hypoplasia with normal visual acuity. Am J Ophthalmol. 1978;86:524-529.

4. Kim RY, Hoyt WF, Lessell S, et al. Superior segmental optic hypoplasia. A sign of maternal diabetes. Arch Ophthalmol. 1989;107:1312-1315.

5. Landau K, Bajka JD, Kirchschläger BM. Topless optic disks in children of mothers with Type I diabetes mellitus. Am J Ophthalmol. 1998;125: 605-611.

6. Hashimoto M, Ohtsuka K, Nakagawa T, et al. Topless optic disk syndrome without maternal diabetes mellitus. Am J Ophthalmol. 1999; 128:111-112.

7. Unoki K, Ohba N, Hoyt WF. Optical coherence tomography of superior segmental optic hypoplasia. Br J Ophthalmol. 2002;86:910-914.

8. Yamamoto T, Sato M, Iwase A. Superior segmental optic hypoplasia found in Tajimi Eye Health Care Project participants. Jpn J Ophthalmol. 2004;48:578-583.

9. Hayashi K, Tomidokoro A, Aihara M, et al. Long-term follow-up of superior segmental optic hypoplasia. Jpn J Ophthalmol. 2008;52: 412-414.

10. Takagi M, Abe H, Hatase T, et al. Superior segmental optic nerve hypoplasia in youth. Jpn J Ophthalmol. 2008;52:468-474.

11. Athappilly G, Pelak VS. Teaching neuroimages: Superior segmental optic nerve hypoplasia confirmed by optical coherence tomography. Neurology. 2009;72:e91-e92.

12. Han SB, Park KH, Kim DM, et al. Prevalence of superior segmental optic nerve hypoplasia in Korea. Jpn J Ophthalmol. 2009;53:225-228.

13. Lee HJ, Kee C. Optical coherence tomography and Heidelberg retina tomography for superior segmental optic hypoplasia. Br J Ophthalmol. 2009;93:1468-1473.

14. Mikelberg FS, Parfitt CM, Swindale NV, et al. Ability of the Heidelberg retina tomograph to detect early glaucomatous visual field loss. J Glaucoma. 1995;4:242-247.

15. Iester M, Mikelberg FS, Drance SM. The effect of optic disc size on diagnostic precision with the Heidelberg retina tomograph. Ophthalmology. 1997; 104:545-548.
16. Wollstein G, Garway-Heath DF, Hitchings RA. Identification of early glaucoma cases with the scanning laser ophthalmoscope. Ophthalmology. 1998;105:1557-1563.

17. Garway-Heath DF, Poinoosawmy D, Wollstein G, et al. Inter- and intraobserver variation in the analysis of optic disc images: Comparison of the Heidelberg retina tomograph and computer assisted planimetry. Br J Ophthalmol. 1999;83:664-669.

18. Swindale NV, Stjepanovic G, Chin A, et al. Automated analysis of normal and glaucomatous optic nerve head topography images. Invest Ophthalmol Vis Sci. 2000;41:1730-1742.

19. Miglior S, Guareschi M, Albe E, et al. Detection of glaucomatous visual field changes using the Moorfields regression analysis of the Heidelberg retina tomograph. Am J Ophthalmol. 2003;136:26-33.

20. Coops A, Henson DB, Kwartz AJ, et al. Automated analysis of Heidelberg retina tomograph optic disc images by glaucoma probability score. Invest Ophthalmol Vis Sci. 2006;47:5348-5355.

21. Medeiros FA, Zangwill LM, Bowd C, et al. Influence of disease severity and optic disc size on the diagnostic performance of imaging instruments in glaucoma. Invest Ophthalmol Vis Sci. 2006;47:1008-1015.

22. Burgansky-Eliash Z, Wollstein G, Bilonick RA, et al. Glaucoma detection with the Heidelberg retina tomograph 3. Ophthalmology. 2007; 114:466-471.

23. Zangwill LM, Jain S, Racette L, et al. The effect of disc size and severity of disease on the diagnostic accuracy of the Heidelberg retina tomograph glaucoma probability score. Invest Ophthalmol Vis Sci. 2007;48:2653-2660.

24. Ferreras A, Pajarín A, Polo V, et al. Diagnostic ability of Heidelberg retina tomograph 3 classifications. Glaucoma probability score versus Moorfields regression analysis. Ophthalmology. 2007;114: 1981-1987.

25. Ferreras A, Pablo LE, Pajarín AB, et al. Diagnostic ability of the Heidelberg retina tomograph 3 for glaucoma. Am J Ophthalmol. 2008; 145:354-359.

26. Ferreras A, Pablo LE, Larrosa JM, et al. Discriminating between normal and glaucoma-damaged eyes with the Heidelberg retina tomograph 3. Ophthalmology. 2008;115:775-781.

27. Kass MA, Heuer DK, Higginbotham EJ, et al. The Ocular Hypertension Treatment Study: A randomized trial determines that topical ocular hypotensive medication delays or prevents the onset of primary open-angle glaucoma. Arch Ophthalmol. 2002;120:701-713.

28. Johnson CA, Sample PA, Zangwill LM, et al. Structure and function evaluation (SAFE): II. Comparison of optic disk and visual field characteristics. Am J Ophthalmol. 2003;135:148-154.
Clinical Ophthalmology

\section{Publish your work in this journal}

Clinical Ophthalmology is an international, peer-reviewed journal covering all subspecialties within ophthalmology. Key topics include: Optometry; Visual science; Pharmacology and drug therapy in eye diseases; Basic Sciences; Primary and Secondary eye care; Patient Safety and Quality of Care Improvements. This journal is indexed on Submit your manuscript here: http://www.dovepress.com/clinical-ophthalmology-journal

\section{Dovepress}

PubMed Central and CAS, and is the official journal of The Society of Clinical Ophthalmology (SCO). The manuscript management system is completely online and includes a very quick and fair peer-review system, which is all easy to use. Visit http://www.dovepress.com/ testimonials.php to read real quotes from published authors. 